written to the Salisbury Unit to tell them how to solve this difficult problem.

Men of science will appreciate this lecture as an example of how to handle an intricate subject with a charm and simplicity of phrase which make it readily intelligible to everybody.

G. LAPAGE

\section{BRITISH ROCKET WEAPONS RESEARCH}

$\mathrm{T}$ HERE is nobody better qualified to discuss our war-time development of rocket weapons than Sir Alwyn Crow, and it would therefore be unprofitable to attempt to dispute the clear statement of facts which he presented as the thirty-fourth Thomas Hawksley Lecture at the Institution of Mechanical Engineers on November 21, 1947, his subject being "The Rocket as a Weapon of War in the British Forces".

Sir Alwyn first traced the development begun in 1935 of the three main types of motor which formed the basis of almost all British rocket-propelled weapons. These were steel tubes of 2 in., 3 in. and 5 in. diameter containing a solid cordite propellant charge, which was ignited at the front, the gases being discharged in each case through a single nozzle at the rear. Pointing out that from the military point of view one of the greatest advantages of the rocket propulsion of missiles is the absence of recoil forces on the projector, Sir Alwyn went on to describe how these motors were combined with different types of explosive head and projection apparatus to meet such varied demands as for anti-aircraft fire, barrage weapons for mass bombardment and airborne projectiles for land and sea targets. He also described the development of rocket motors of the high degree of reliability necessary to provide the extra thrust which enabled aircraft to take off with a much shorter run. An idea of what was achieved in these fields may be gathered from the fact that "a single craft was able to fire highly lethal ammunition on the chosen target area at the rate of half a ton a minute for nearly a minute at a time", and the hitting power of a single aircraft was "momentarily the equivalent of the full firing power of a medium cruiser".

As this was his main concern, Sir Alwyn's paper dealt primarily with the detailed development of solid-fuelled rockets to meet a number of specific requirements; but since there can be little doubt that British progress in the field of rocket development at the end of the War was some ten years behind that of the Germans, it would be interesting if Sir Alwyn could be persuaded to present his views on a number of the other aspects of this type of armament, aspects which must have been the subject of much discussion because of their fundamental nature. Were difficulties of production and the impossibility of making the necessary research in the early stages the obstacles that precluded the use of liquid fuels, with the enhanced index of performance which would be expected from them ? Why was so much greater emphasis placed on fin stabilization than on spin stabilization? Was progress made in the examination of methods of control and guidance of the projectile during its flight, or was it considered in Britain that the inherent advantages of the unguided weapon outweighed any which might accrue from guidance ? Even our choice of solid propellants appears to have been very limited, and it would be interesting to know what attempts were made to develop other types. The use of light alloy tubing for the motor body would seem to be an obvious way to increase the charge/weight ratio, and the steps made to investigate its possibilities would be worth knowing.

The only fair criticism which can perbaps be made of Sir Alwyn's lecture is that he made no mention of the aiming and sighting equipment developed to ensure that the projectiles would arrive at the right spot at the right moment, for if they fail to do this all the ingenuity lavished on the design of the weapon is useless.

R. H. MACMILLAN

\section{PHOTO-ELASTICITY IN MECHANICAL ENGINEERING}

$T$ WO papers which make a very useful addition to the sparse literature on photo-elasticity were presented to the Institution of Mechanical Engineers on December 12, 1947. Mr. W. A. P. Fisher's paper dealt with the basic physical properties of model materials which are relied upon in the 'frozen stress' technique of three-dimensional stress analysis. This technique, which was originated in Germany and the United States, is based on the fact that certain photo-elastic model materials have a very low Young's modulus at elevated temperatures and consequently suffer considerable deformations under small loads. If such small loads remain in position during subsequent cooling, it is found that the materials set in the strained position and retain, after the removal of the loads, virtually the whole of the strain and the photo-elastic effect associated with the loading at the elevated temperature; hence the term 'frozen stress'. This phenomenon is utilized in three-dimensional analysis by cutting thin slices from a threedimensional model, which can then be examined in a photo-elastic polariscope in accordance with the ordinary two-dimensional technique. The 'frozen stress' patterms are not found to be appreciably disturbed by careful cutting.

The major part of Mr. Fisher's paper was devoted to a discussion of the suitability of phenol-formaldehyde resins, particularly 'Catalin 800 ', for use with this technique. In the United States, glyptal resins have been used for this purpose almost without exception but as this material is very expensive and difficult to obtain in Britain, considerable interest is attached to the detailed investigation of the merits of 'Catalin' which was carried out at the Royal Aircraft Establishment, Farnborough, by Mr. Fisher. Data were given in the paper of the physical properties of 'Catalin', both at room temperature and at its softening temperature. Young's modulus at $80^{\circ} \mathrm{C}$. appears to be about $1 / 120$ of the value at room temperature. The paper also contained some practical illustrations of the 'freezing' technique.

Mr. R. B. Heywood's paper on "Some Modern Applications of Photo-elasticity" contained a brief outline of photo-elastic theory and a summary of the properties of the more important model materials, followed by some interesting examples of applications to design problems taken from the field of mechanical engineering.

It will be an encouragement to everyone interested in photo-elasticity that the engineering institutions are once more, after a long interval, taking an interest in this valuable method of stress analysis.

E. K. FrankL 